\title{
The sPHENIX Compact Time Projection Chamber: Study of SpaceCharge Distortions from First Principles
}

Carlos E. Pérez Lara*, for the sPHENIX collaboration Stony Brook University, NY, U.S.A.

E-mail: carlos.perezlaraestonybrook.edu

The upcoming sPHENIX experiment [1] will have a compact Time Projection Chamber (TPC) as a main central $(-1.1<\eta<+1.1)$ tracking system. The TPC will work in continuous readout mode at $15 \mathrm{kHz}$, although the collision rate delivered by RHIC at $\pm 30 \mathrm{~cm}$ could reach up to 100 $\mathrm{kHz}$. Spacecharge stockpile is one of the important challenges to overcome in such conditions. The present article describes a study on the amount of distortions expected due to spacecharge in the sPHENIX TPC volume under the expected running conditions.

5th International Conference on Micro-Pattern Gas Detectors (MPGD2017)

22-26 May, 2017

Philadelphia, USA

${ }^{*}$ Speaker. 


\section{Introduction}

Ionization trails are formed in the TPC gas volume as charged particles travel through it. These trails are formed as a combination of positive ions and secondary electrons. The electrons produced travel quickly towards the collection (anode) plates and in their way they collide with gas molecules causing them to deflect in the X-Y plane. This process is called diffusion and its underlying dynamics is effectively modeled by a random walk with one longitudinal and one transverse coupling coefficient. The diffusion coefficients depend solely on the gas mixture and on the electric and magnetic fields in which the electron motion takes place.

On the other hand, the (massive) ions move slowly towards the central membrane. Since the collision rate is high, there is a chance that a second collision takes place before the ions are completely gone. Additionally, there is a second source of ions injected back from the GEM amplification chain in the anode plate called Ion Back Flow (IBF). If not stopped, these back flow ions will travel the full distance between the end plates and the central membrane at terminal speed. The amount of IBF depends on the overall amplification of the GEM configuration and typical values are of the order of $1 \%$ IBF for a 2000 gain.

\section{Simulation of Charge Density Accretion}

When a collision takes place, the charged particles are allowed to deposit energy in the TPC gas. The energy is in turn translated into electrons from the number of effective ionization electrons produced per $\mathrm{keV}$ in the gas mixture under consideration (figure 1). The electrons (ions) are let stream free towards the collection plates (central membrane) for $\Delta \tau$ time. If the electrons arrived to the collection plate before $\Delta \tau$, which is most of the time for the collision rates considered, ions from IBF are injected and propagated towards the central membrane. When the ions touch the central membrane, they are removed from the gas volume (left side of figure 2).

The rates at RHIC were simulated sampling event by event a time in between collisions $\Delta \tau$ from a poissonian distribution whose mean represents different collision rates scenarios: $15 \mathrm{kHz}$, $25 \mathrm{kHz}, 50 \mathrm{kHz}$ and taking into account a minimal time unit of $106 \mathrm{~ns}$, which corresponds to RHIC's beam structure. Figure 2 shows the resulting charge density for our gas with $1 \%$ IBF at $15 \mathrm{kHz}$.
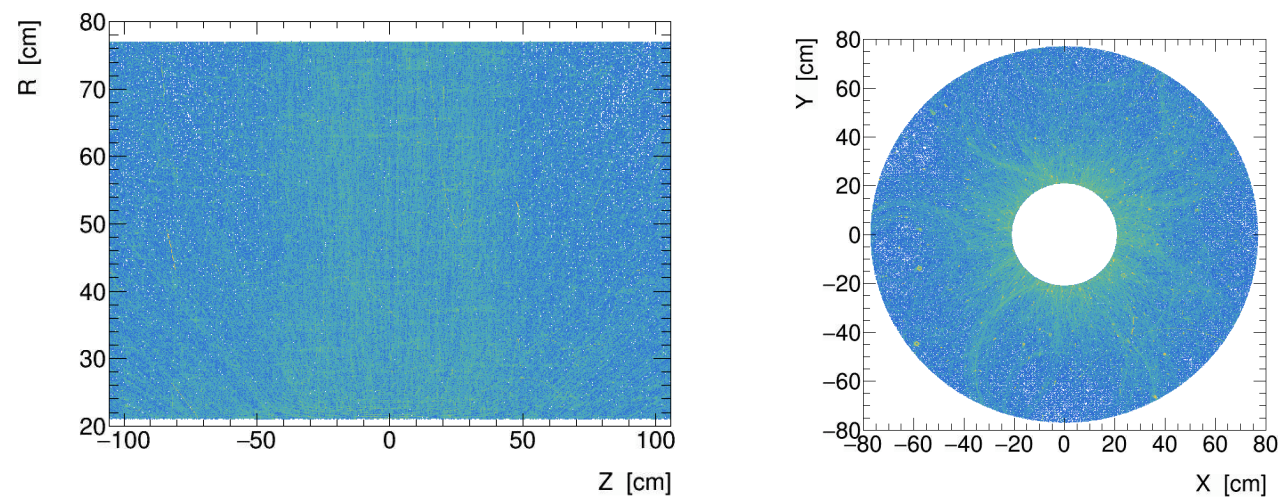

Figure 1: Ion density profiles for one Au+Au@200 GeV Hijing+Geant4 event generated in the Ne-based gas volume of the sPHENIX TPC. 

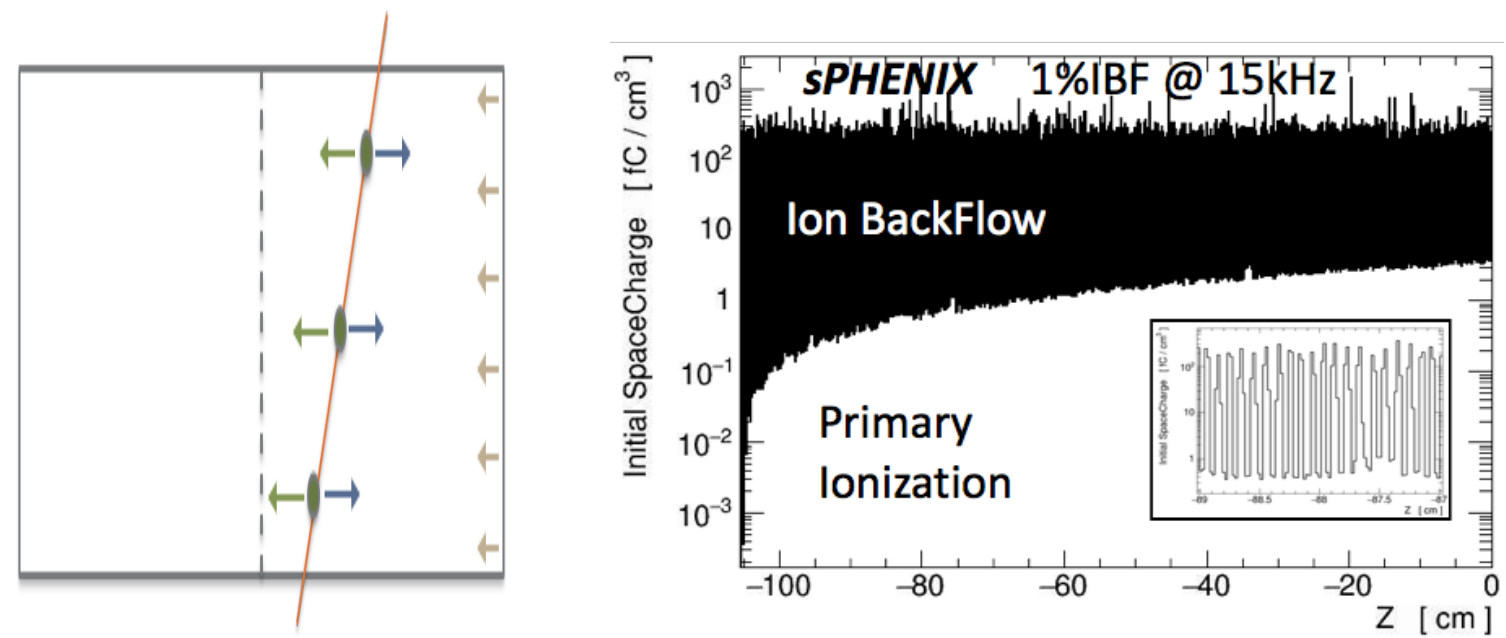

Figure 2: Simulation of spacecharge density after several Au+Au MB collisions at $\sqrt{\mathrm{s}}=200 \mathrm{GeV}$ showing the two components present. The ions injected back from GEM back flow is the driving source of spacecharge. The inset shows clearer the circular structure obtained from the collector.

The particles abundance and kinematics used here come from central Au+Au Hijing events for collisions at $200 \mathrm{GeV}$ plus Geant4 in order to model the energy deposition and tracking in the magnetic field. The material budget of all sPHENIX detectors [2] were included in the simulation.

\section{Distortions in the Electric Field due to Spacecharge}

The electric field produced due to spacecharge stockpile can be computed analytically using Green's function to solve the problem of a point charge inside a cylindrical grounded vessel. The electric field due to spacecharge for every point inside the cage can then be computed as

$$
E(r, \varphi, z)=-\nabla \Phi(r, \varphi, z), \quad \Phi(r, \varphi, z)=\frac{1}{\varepsilon_{0}} \int_{V^{\prime}} r^{\prime} d r^{\prime} d \varphi^{\prime} d z^{\prime} \rho\left(r^{\prime}, \varphi^{\prime}, z^{\prime}\right) \cdot G\left(r, \varphi, z, r^{\prime}, \varphi^{\prime}, z^{\prime}\right)
$$

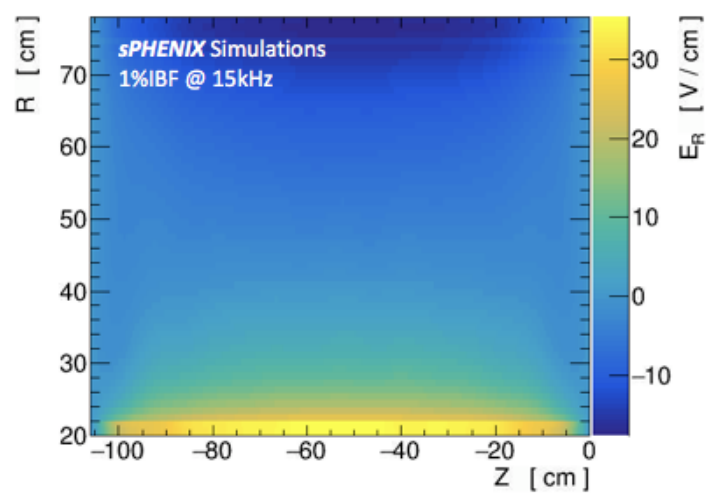

Figure 3: Radial component of the electric field generated solely by spacecharge for the TPC volume in between the anode and catode planes. 

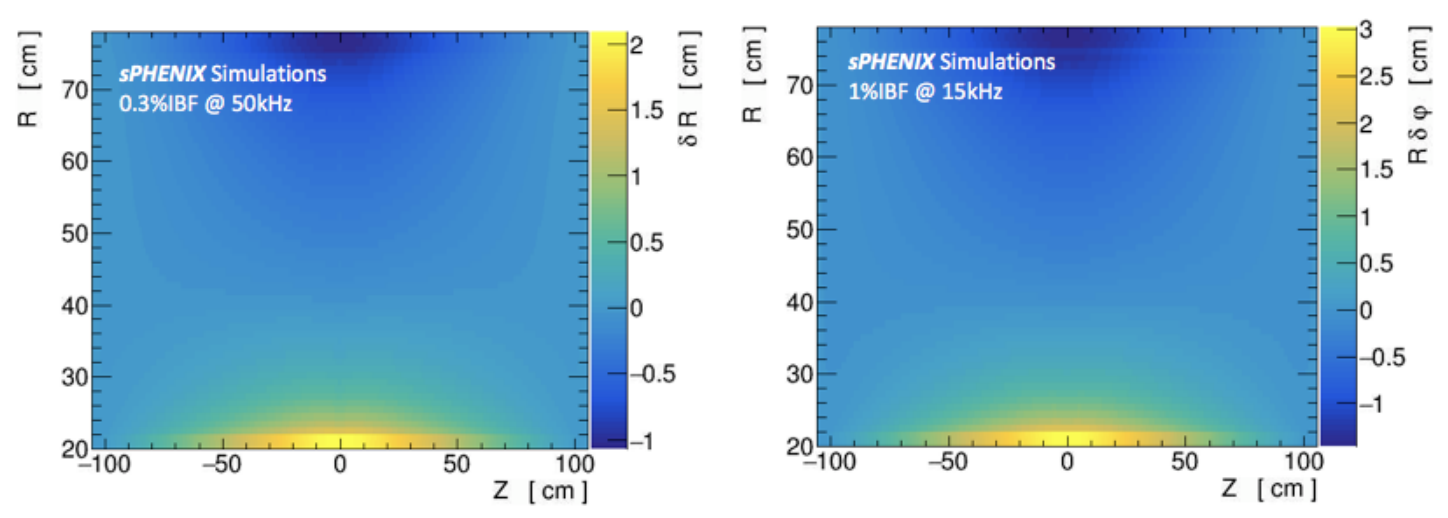

Figure 4: Radial and azimuthal distortions associated to two different configuration for ion back flow percentage and colliding frequency. The distortions are maximal close to the cylindrical enclosure and central membrane.

where the integral is over the whole volume confined by the TPC cage. This problem has been extensively studied for ALICE's TPC [3], where it was found that the solution can be written as an expansion in the Bessel base. For this work a truncation to the 15th order was found to converge for the dimensions of the sPHENIX TPC.

\section{Distortions in Traveled Path from Generation to Readout}

Single electrons drifting under the presence of electric and magnetic fields can be modelled using the Langevin equation [4]:

$$
m \frac{d \vec{u}}{d t}=q \vec{E}+q[\vec{u} \times \vec{B}]-K \vec{u},
$$

where $\vec{u}$ is the drift velocity, $\mathrm{q}$ is the charge of the drifting particle in units of electron charge, the first two terms at the right hand side of the equation account or the electric force while the third is an effective frictional force which is related to the mean free path in the gas $(K>0)$. The steady solution $\left(\frac{d \vec{u}}{d t}=0\right)$ under adiabatic approximation $\left(t \gg \frac{m}{K}\right)$ is

$$
\vec{u}=\frac{\mu|\vec{E}|}{1+\omega^{2} \tau^{2}}\left[\hat{E}+\omega \tau(\hat{E} \times \hat{B})+\omega^{2} \tau^{2}(\hat{E} \cdot \hat{B}) \hat{B}\right], \quad \delta_{r}=\int_{s} u_{r} d t=\int_{s} \frac{u_{r}}{u_{z}} d z
$$

where $\mu=\frac{q}{K}$ is the ion mobility in the gas, $\omega$ is the cyclotron frequency for the electron and $\tau$ is the mean interaction time between drifting electrons and atoms in the gas. $\omega \tau=q \mu|\vec{B}|$.

The spatial distortion profiles are shown in figure 4 . The results show that the maximal distortions are obtained close to the field cage and central membrane. The distortions found are of the order of few centimeters which is one order of magnitude less than those found for the ALICE case. When it comes to distortions, two geometrical properties compete with each other as aggravators: the proximity of the cage to the interaction point and the long extension in Z. Notice also that as a function of $\mathrm{R}$, both the $\delta R$ and $R \delta \varphi$ change sign when moving from the inner to the outer cage wall.

Figure 5 shows a one axis projection of the $\delta R$ and $R \delta \varphi$ distortions for several IBF and collisional rate configurations. Distortions are found to increase monotonically as a function of these 

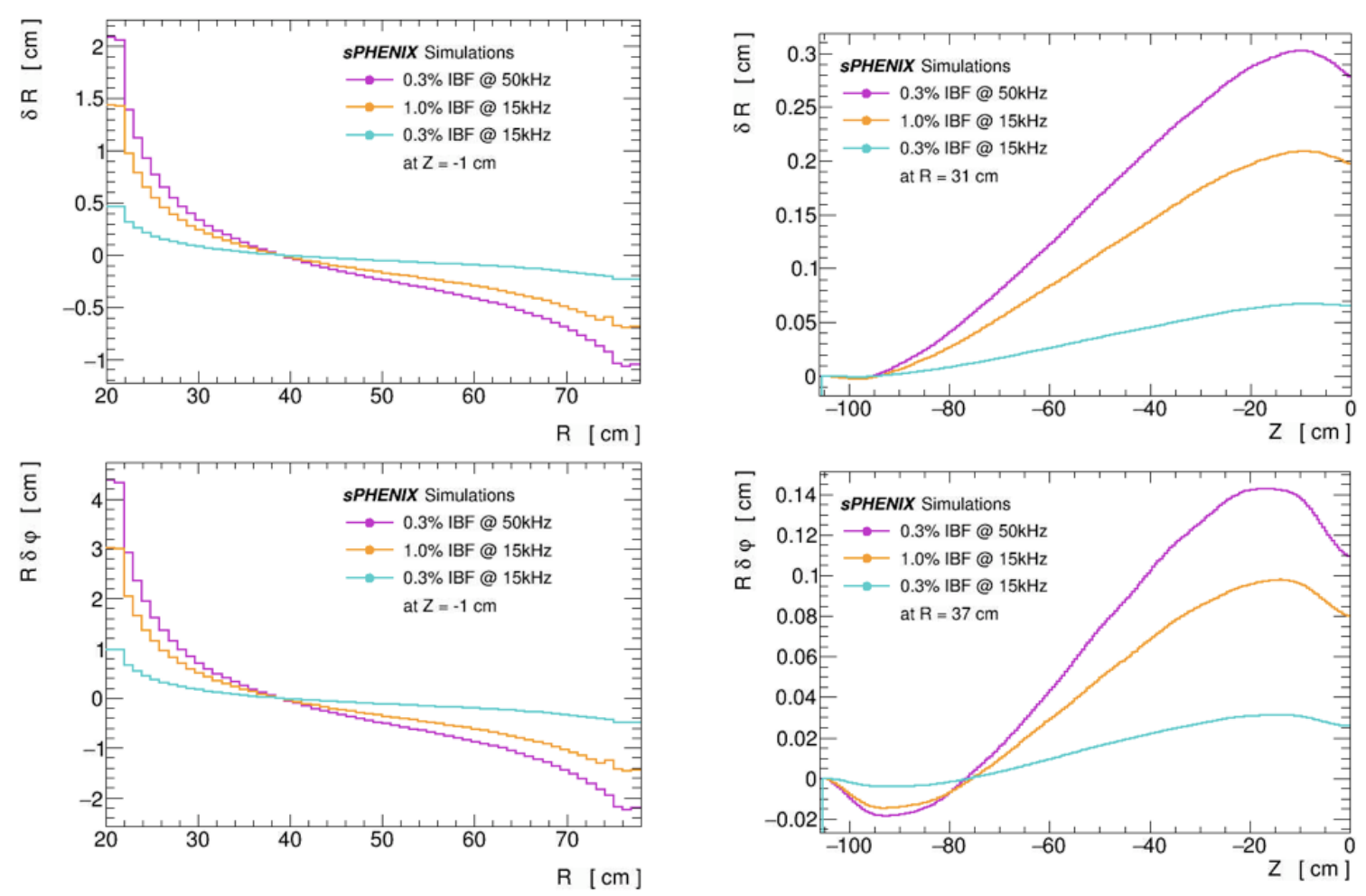

Figure 5: Radial (top) and azimuthal (bottom) distortions produced in the sPHENIX TPC for different collision rates and different IBF percentages as a function of $\mathrm{R}$ in the left panels and $\mathrm{Z}$ in the right panels.

two parameters. For all the cases explored here the distortions are expected in the order of few centimeters at most.

\section{Acknowledgments}

I would like to extend my gratitude to Jens Wiechula, Harald Appelshaeuser, Marian Ivanov and Jim Thomas for the email exchanges that led to the realization of this work. The complete code used for this simulation is a standalone package which can be found in [5].

\section{References}

[1] Adare, A. and others, An Upgrade Proposal from the PHENIX Collaboration, ARXIV:1501.06197

[2] Pérez Lara, Carlos E., The sPHENIX Experiment, EPJ Web Conf. 17110002 (2018) DOI: 10.1051/epjconf/201817110002

[3] S. Rossegger, Simulation and Calibration of the ALICE TPC Including Innovative Space Charge Calculations, PhD thesis, University of Technology, Graz, 2009, CERN-THESIS-2009- 124.

[4] M. Mager, S. Rossegger and J. Thomas The Langevin equation expanded to 2nd order and comments on using the equation to correct for space point distortions in a TPC, ALICE-INT-2010-016.

[5] Pérez Lara, Carlos E., A minimal model to study spacecharge distortions due to ion-back-flow in GEM-based TPC under continuous readout,

https://github.com/carlosperezlara/TPCSpaceChargeDistortion.git 